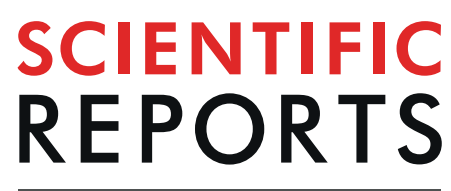

natureresearch

\title{
Glycofullerenes as non-receptor tyrosine kinase inhibitors- towards better nanotherapeutics for pancreatic cancer treatment
}

\author{
Maciej Serda ${ }^{1 *}$, Katarzyna Malarz ${ }^{2}$, Anna Mrozek-Wilczkiewicz ${ }^{2}$, Marcin Wojtyniak $^{2}$, \\ Robert Musioł ${ }^{1} \&$ Steven A. Curley ${ }^{3}$
}

The water-soluble glycofullerenes GF1 and GF2 were synthesized using two-step modified BingelHirsch methodology. Interestingly, we identified buckyballs as a novel class of non-receptor Src kinases inhibitors. The evaluated compounds were found to inhibit Fyn A and BTK proteins with $\mathrm{IC}_{50}$ values in the low micromolar range, with the most active compound at $39 \mu \mathrm{M}$. Moreover, we have demonstrated that formation of protein corona on the surface of [60]fullerene derivatives is changing the landscape of their activity, tuning the selectivity of obtained carbon nanomaterials towards Fyn A and BTK kinases. The performed molecular biology studies revealed no cytotoxicity and no influence of engineered carbon nanomaterials on the cell cycle of PANC-1 and AsPC-1 cancer cell lines. Incubation with the tested compounds resulted in the cellular redox imbalance triggering the repair systems and influenced the changing of protein levels.

Pancreatic cancer is an extremely aggressive type of cancer with a poor prognosis and a five-year survival rate of less than $7 \%^{1}$. Moreover, only about $20 \%$ of patients are eligible for surgical resection, the only potentially curative treatment ${ }^{2}$. Chemotherapeutic approaches, which are often based on gemcitabine, are still present in the clinic as the adjuvant therapy of choice for resected pancreatic cancer. The novel cytotoxic combination strategies and nanoformulations which include Folfirinox and nab-paclitaxel are being developed intensively, but these treatments have their own shortcomings and their therapeutic effects are disappointing ${ }^{3}$. Nanomedical strategies which include metallofullerene-based $\left(\mathrm{Gd} @ \mathrm{C}_{82}(\mathrm{OH})_{22}\right)$ inhibitors of matrix metalloproteinases (MMPs) ${ }^{4}$, photothermally active nanoparticles ${ }^{5,6}$, and several nanodelivery systems $\mathrm{s}^{7,8}$ are also being presented in attempts to improve survival in pancreatic cancer patients. At the same time, the pharmaceutical industry has focused significant attention around the use of selective tyrosine kinase inhibitors as anti-cancer agents ${ }^{9}$. The EGFR kinase inhibitor Erlotinib has been approved by FDA for use in combination with gemcitabine for locally advanced or metastatic pancreatic cancer ${ }^{10}$. Recently, some reports have suggested that inhibition of non-receptor intracellular Src kinases inhibits growth and metastasis of human pancreatic carcinoma in murine models, and is followed by synergetic effects in combination therapy with gemcitabine ${ }^{11}$. In order to overcome the barriers to standard chemotherapeutics, we have been exploring the use of [60]fullerene derivatives as novel EPR-targeted nanotherapeutics in in vitro and in vivo models ${ }^{12,13}$. Previously, we have reported the creation of photoactive and highly water-soluble glycofullerene GF2 (termed 'Sweet- $\mathrm{C}_{60}$ ') that predominantly accumulates in the nucleus of pancreatic stellate cells (PSCs) and is inherently non-toxic even in high concentrations (above $1 \mathrm{mg} / \mathrm{mL})^{14}$.

\section{Results and Discussion}

Based on the above background, we have synthesized two glycofullerenes: GF1 and GF2 (see Fig. 1A) containing $D$-glucosamine fragments as glycosyl donors in the central core, including full characterization of all intermediates through MALDI-TOF mass spectroscopy, ESI-mass spectroscopy, ${ }^{1} \mathrm{H}$ - and ${ }^{13} \mathrm{C}-\mathrm{NMR}$, DLS, and zeta potential measurements (see Supporting Information, Figs. S1-S5). The Src kinase inhibitory properties for the synthesized

${ }^{1}$ Institute of Chemistry, University of Silesia in Katowice, Katowice, 40-006, Poland. ${ }^{2}$ Institute of Physics and Silesian Center for Education and Interdisciplinary Research, University of Silesia in Katowice, 75 Pułku Piechoty 1A, Chorzów, 41-500, Poland. ${ }^{3}$ CHRISTUS Trinity Mother Frances Oncology Institute, Tyler, TX, 7570, USA. *email: maciej.serda@us.edu.pl 
A

B

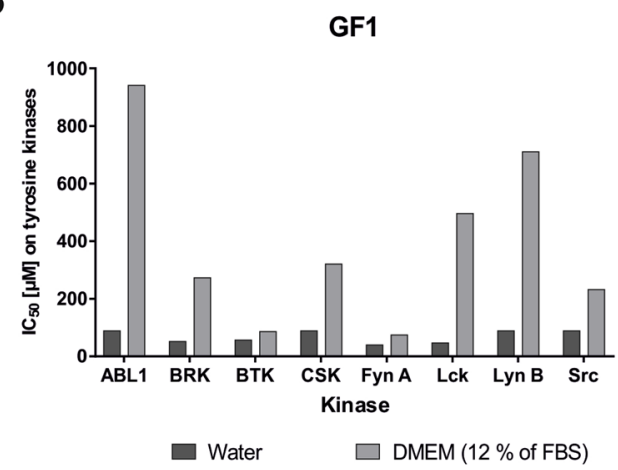

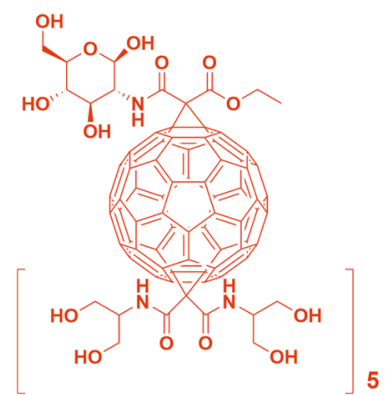

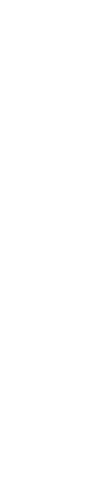

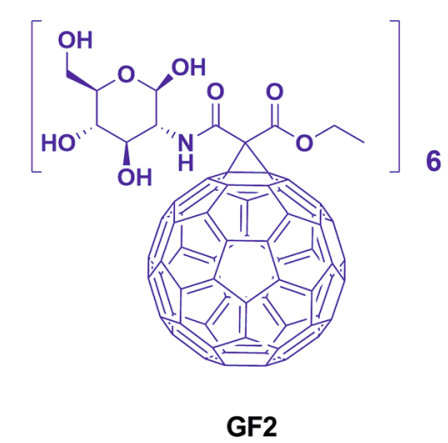

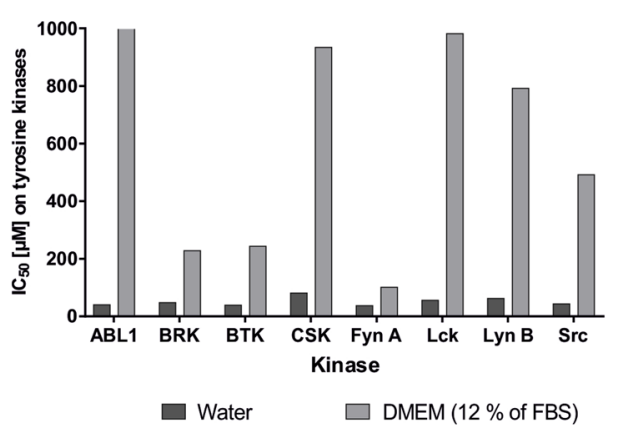

Figure 1. (A) Structures of GF1 and GF2 glycofullerenes; (B) Inhibitory activity of glycofullerenes on a panel of non-receptor tyrosine kinases.

glycofullerenes have been thoroughly evaluated, and detailed studies on protein corona formation around the glycofullerenes and its influence on selectivity and inhibitory Src kinase activity (see Fig. 1B and Table 1) have been carried out. The enzymatic studies of [60]fullerenes have been extended to time-dependent mRNA and protein expression analysis for Fyn A and Lck kinases which was performed on two human pancreatic cancer cell lines: PANC-1 and AsPC-1. The influence of glycofullerenes on cell cycle regulation as well as induction of autophagy in pancreatic cancer cell lines have also been investigated. To the best of our knowledge, this is the first work on the use of [60]fullerene nanomaterials as selective non-receptor tyrosine kinases inhibitors.

Previously, we have demonstrated that fluorescently-labelled [60] fullerene derivatives were able to extravasate more into orthotopic murine breast tumor tissues than into the contralateral mammary fat pad ${ }^{13}$. In this study we propose that glycosylation of the [60]fullerene core will promote cancer targeting of engineered carbon nanomaterials due to overexpression of glucose transporter membrane proteins (GLUTs) in several cancers, including pancreatic adenocarcinoma ${ }^{15}$. Interestingly, we have performed qRT-PCR experiments on two human pancreatic cancer cell lines, showing that AsPC-1 cells present 10 fold higher levels of GLUT-1 mRNAs in comparison to PANC-1 cells (see Supporting Information, Fig. S9). We decided to use diserinol malonate (possessing four hydroxyl group) as the second addend attached to our [60]fullerene scaffold. Our previously published experiments describing molecular biology impact of compound $\mathrm{C}_{60}$-ser have reported its highly water-soluble properties and lack of in vitro cellular and in vivo murine toxicity ${ }^{16,17}$. Moreover, the metal chelating abilities of hydroxyfullerenes have been recently explored, and they should be considered as an important factor modulating their interactions with cellular targets, such as enzymes ${ }^{18}$.

To synthesize the aforementioned structurally diverse glycofullerenes, the modified Bingel-Hirsch methodology was applied. The presented synthetic procedure enables the creation of a broad spectrum of [60]fullerenes and allows generation of structurally more complicated systems thanks to high synthetic accessibility of functionalization malonates. The [60]fullerene nanomaterials GF1 and GF2 were synthesized in the two-step cyclopropanation reactions, in which [60]fullerene derivative 3 was used as the substrate for the final products (see Supporting Information, Fig. S1). The [60]fullerene hexakis-adduct with $T_{h}$-symmetry (GF1) bearing two or more different peripheral functional subunits was obtained in the second Bingel reaction from monoadduct 3 as a starting material. The glycofullerene GF2 was obtained as a mixture of regioisomers without further purification, and its full chemical characterization had been published earlier ${ }^{14}$. The MALDI-TOF mass spectrum of peracetylated GF1 is shown in Fig. S7 (Supporting Information), showing characteristic molecular peaks for hexakis-adduct at $3412 \mathrm{Da}$. A molecular peak ion from the deprotected sodium adduct GF1 is presented at 2273.2 Da, showing also small signals from pentakis isomers at 2026.1 Da (Supporting Information, Fig. S8). As can be seen in Fig. S4 (Supporting Information), glycofullerene GF1 seems to form aggregates at around $100 \mathrm{~nm}$ and shows a surface charge of $-26.7 \mathrm{mV}$ based on DLS and zeta potential measurements. Mackeyev et al. reported experiments conducted for the water-soluble $\mathrm{C}_{60}$ derivatives, especially those with diserinol units, suggested that those derivatives form highly-dynamic aggregates, sized between $100-300 \mathrm{~nm}$ in diameter, which exist in equilibrium with free, hydrated fullerene molecules (based on SEM studies) ${ }^{19,20}$. Moreover this equilibrium is connected with addition of organic solvents and ionic strength (salts), which complicates understanding of protein corona formation. In addition, to better understand formation of glycofullerene aggregates, we performed AFM studies using the $\mathrm{Si}$ 


\begin{tabular}{|c|c|c|c|c|c|c|c|c|}
\hline \multirow[b]{2}{*}{ Compound } & \multicolumn{8}{|c|}{$\mathrm{IC}_{50}[\mu \mathrm{M}]$ on tyrosine kinases } \\
\hline & ABL1 & BRK & BTK & CSK & Fyn A & Lck & Lyn B & Src \\
\hline GF1 in water* & $>88.850$ & $51.533 \pm 9.329$ & $56.864 \pm 12.439$ & $>88.850$ & $39.982 \pm 2.665$ & $46.645 \pm 3.554$ & $>88.850$ & $>88.850$ \\
\hline $\begin{array}{l}\text { GF1 in } \\
\text { DMEM* }\end{array}$ & $940.91 \pm 149.711$ & $272.768 \pm 46.646$ & $86.184 \pm 6.664$ & $320.302 \pm 53.310$ & $74.189 \pm 20.88$ & $496.224 \pm 20.880$ & $710.351 \pm 139.494$ & $231.897 \pm 46.202$ \\
\hline GF2 in water* & $40.146 \pm 2.028$ & $47.851 \pm 3.244$ & $38.929 \pm 3.244$ & $>81.103$ & $37.307 \pm 3.244$ & $55.961 \pm 11.760$ & $62.044 \pm 1.216$ & $43.796 \pm 1.216$ \\
\hline $\begin{array}{l}\text { GF2 in } \\
\text { DMEM* }\end{array}$ & $>1000$ & $228.305 \pm 60.827$ & $243.714 \pm 38.118$ & $933.901 \pm 229.16$ & $100.973 \pm 17.437$ & $981.346 \pm 221.4112$ & $791.565 \pm 238.037$ & $491.484 \pm 156.123$ \\
\hline Dasatinib $^{21 * *}$ & $0.0031 \pm 0.0005$ & $0.005 \pm 0.0005$ & $0.003 \pm 0.0007$ & $0.0013 \pm 0.0003$ & $0.0016 \pm 0.0008$ & $0.0023 \pm 0.0007$ & $0.0012 \pm 0.0001$ & $0.0009 \pm 0.0001$ \\
\hline
\end{tabular}

Table 1. Inhibitory activity of glycofullerenes GF1 and GF2 on non-receptor tyrosine kinases panel performed in water and in DMEM with $12 \%$ of FBS. *The final concentration of solvents (water, DMEM or DMSO) is $5 \%$. **Dasatinib was dissolved in DMSO.

(001) substrate. As one can notice, the substrate was extremely flat, with the roughness, calculated as root mean square (RMS), of only $72.8 \mathrm{pm}$ (Fig. S6A). The topography obtained from the AFM shows two types of agglomerates for both glycofullerenes GF1 and GF2. In the case of GF1, the first type consist of separate round-like objects, with the average diameter equal to $19.24 \mathrm{~nm}$ diameter on average (Fig. S6B), b) larger agglomerates with average sizes of 270-300 nm (Fig. S6C). The [60]fullerene derivative GF2 shown in Figs. S6D-E was found to be very similar to the previous sample. We have observed two primary types of agglomerates- smaller and larger ones. The primary agglomerates were found to have $17.68 \mathrm{~nm}$ diameter on average and heights around $10 \mathrm{~nm}$, while the larger ones were around $250-280 \mathrm{~nm}$ in diameter and height of $400 \mathrm{~nm}$ consisting of smaller objects. The smaller observed objects were often asymmetric, suggesting that they are formed of even smaller parts (separate fullerenes). Unfortunately, due to the size of the AFM tip limiting the image resolution, we were not successful in observing those fullerenes. The larger objects however, could be easily distinguished, and were formed of smaller agglomerates. Due to protein corona formation on the surface of synthesized glycofullerenes, we were also interested in the samples dissolved in the DMEM containing FBS, however we have found a huge difficulties in the measurements of those samples. The typical result for the FBS containing sample is shown in Fig. S6E. One can notice very large structures (tens of $\mu \mathrm{m}$ in diameter), with branch- or leaf-like morphology. Unfortunately, the structures were so large, that no glycofullerenes were detected. We have performed a number of experiments with diluted samples trying to limit the salt content. The crystal sizes could be diminished, but still no aggregates were visible. This could be due to the fact that those aggregates could serve as perfect base for the salt crystals to growth from, thus hiding the agglomerates inside.

With a synthetic methodology in hand, we have tested the [60]fullerene nanomaterials as potential inhibitors of non-receptor tyrosine kinases including the ABL1, BRK, BTK, and Src family kinases (CSK, Fyn A, Lck, Lyn $\mathrm{B}$ and $\mathrm{Src}$ ). As depicted in Table 1, the GF1 compound exhibits inhibitory properties in micromolar ranges, modulating the catalytic activity of Fyn A and Lck proteins ( $\mathrm{IC}_{50}$ parameters 39.982 and $46.645 \mu \mathrm{M}$, respectively). Interestingly, almost no inhibitory activity was observed for the ABL1, CSK, Lyn B, and Src kinases. The GF2 compound shows a relatively high inhibitory activity pattern for Src family kinases, with the exception of the CSK protein, and it also presents the lowest value of the $\mathrm{IC}_{50}$ parameter for the Fyn A enzyme $(37.307 \mu \mathrm{M})$. Additionally, this carbon nanomaterial exhibits a high potential of inhibition against the ABL1, BRK, and BTK kinases. Due to the lack of water-soluble nanomaterials with inhibitory activity on the Src kinases, a small molecular inhibitor dasatinib has been used as a reference compound in these studies. It presents a nanomolar activity on all tested tyrosine kinases, but possesses a low selectivity profile within the tested subgroup of kinases, as well as cytotoxic effects on a normal cell line (NHDF) ${ }^{21}$.

It has been previously reported by experimental and computational approaches that at least 30 proteins may interact with [60]fullerenes, including human serum albumins, lysozyme, and HIV proteases ${ }^{22}$. Based on plethora of literature reports describing formation of protein corona on the surface of carbon nanomaterials, we have decided to carry out inhibition experiments using a cellular medium DMEM containing $12 \%$ of fetal bovine serum $(\mathrm{FBS})^{23}$ as a solvent. The DLS measurements (see Supporting Information, Fig. S5) on the GF1 glycofullerene confirm that it forms aggregates of around $100 \mathrm{~nm}$ that are being rapidly reorganized in the presence of FBS into three different subgroups: 8,30 , and $195 \mathrm{~nm}$. The final results of inhibitory activity of $\mathrm{C}_{60}$-FBS complexes are depicted in Table 1. As expected, the protein corona formed on the surface of glycofullerenes have modified the landscape of their reactivity changing it to higher values of $\mathrm{IC}_{50}$ parameters (Fig. 1B). What is more appealing, the selectivity profiles of GF1 and GF2 have been significantly altered. For the GF1 compound, it is inactive towards almost all the tested tyrosine kinases with the exception of the Fyn A and BTK kinases with $\mathrm{IC}_{50}$ parameters below $100 \mu \mathrm{M}$. The GF2 glycofullerene shows the greatest inhibitory activity on Fyn A kinase ( $\mathrm{IC}_{50}$ parameter around $\left.100 \mu \mathrm{M}\right)$ with no activity on ABL1, CSK, Lck, and Lyn B. Although molecular docking studies should also be performed in order to analyze the $\mathrm{C}_{60}$-kinases interactions, it can be assumed that glycofullernes do not interact with the active site of the aforementioned kinases but rather show an allosteric type of inhibition.

To better understand interactions of fullerene nanomaterials with serum proteins, as well as their inhibitory activities on non-receptor kinases, we incubated compound GF2 at two different concentrations ( 1 and $2 \mathrm{mg}$ per $\mathrm{mL}$ ) with $10 \%$ and $100 \%$ of FBS. The results are depicted on the Fig. S11 in the Supporting Information. Based on the densitometric analysis, we could confirm that the intensities of protein bands around 60,110 and $160 \mathrm{kDa}$ are changed (in comparison to $100 \%$ of FBS alone), what is related to the concentration of [60]fullerene nanomaterial. Interestingly, when GF2 is incubated with FBS at higher concentration $(2 \mathrm{mg} / \mathrm{mL})$, the most increased band 
A

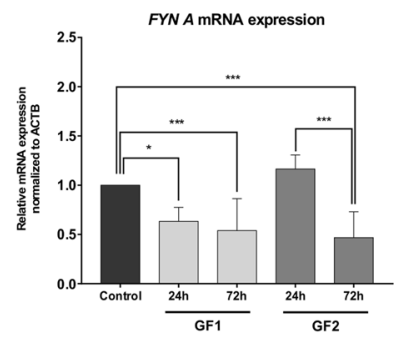

PANC-1 cells

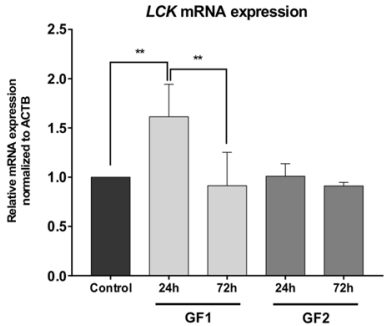

B

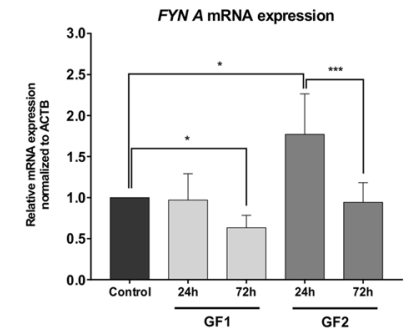

AsPC-1 cells

Figure 2. qRT-PCR analysis of expression of FYN and LCK in PANC-1 (A) and AsPC-1 cells (B) after 24hour and 72-hour incubation with glycofullerenes $(1 \mathrm{mg} / \mathrm{mL})$. The results are shown as the mean $\pm \mathrm{SD}$ of four independent measurements, each in triplicate. Data were analyzed using one-way ANOVA with Bonferroni's post-hoc test: $* \mathrm{p}<0.05, * * \mathrm{p}<0.01, * * * \mathrm{p}<0.001$ compared to the control and respective compounds.

A

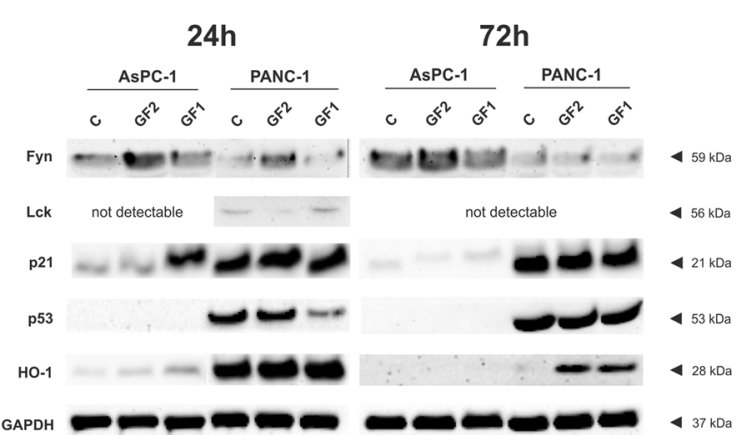

B
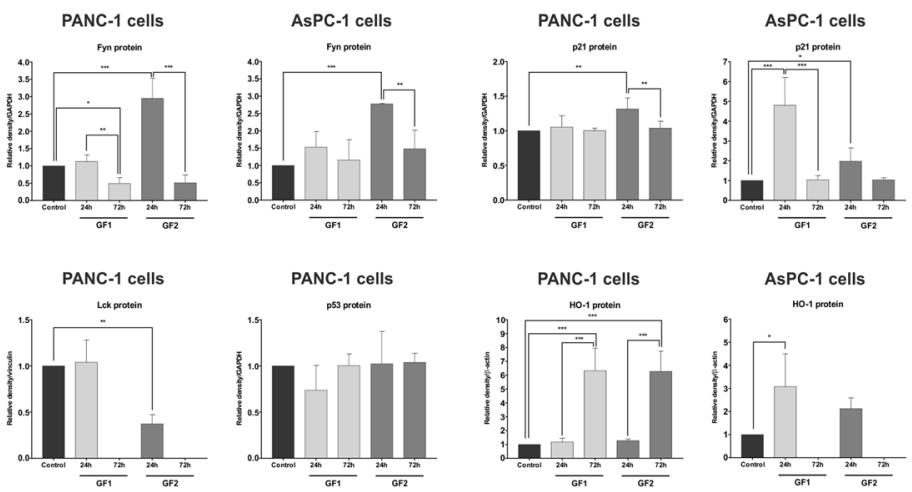

Figure 3. The effect of glycofullerenes $(1 \mathrm{mg} / \mathrm{mL})$ on the expression of Fyn, Lck, p21, p53, and HO-1 in PANC-1 and AsPC-1 cells (A). Densitometric analyses of these proteins were normalized to GAPDH, vinculin, or $\beta$-actin. The results are the mean \pm SD of three independent experiments. Statistical differences were analyzed using one-way ANOVA with a Bonferroni's post-hoc test: ${ }^{*} \mathrm{p}<0.05,{ }^{*} \mathrm{p}<0.01,{ }^{*} * \mathrm{p}<0.001$ compared to the control group and respective compounds (B).

is that around $65 \mathrm{kDa}$ (it could be associated as bovine serum albumin band, molecular weight around $66 \mathrm{kDa}$ ). In contrast for the lower concentration studies, one could observe marked increasement of bands for the higher mass proteins $(110$ and $160 \mathrm{kDa}$ ). This phenomenon is also observed when the $10 \%$ percent FBS studies, and also confirmed by other reports in which different nanomaterial concentrations could affect formation of different protein coronas ${ }^{24}$. The obtained experimental results support the hypothesis of protein corona formation around synthesized glycofullerene GF2, but further mass spectrometry followed bioinformatic analysis should be carried out to identify the exact binding proteins.

The interesting ability of glycofullerenes to inhibit tyrosine kinases in vitro prompted us to evaluate their mechanism of action at the cellular level. For this purpose, we have performed an analysis of the transcript and the protein levels of Fyn and Lck in the two human pancreatic cell lines after treatment with these glycofullerenes. The results of these experiments are presented in Figs. 2 and 3. The qRT-PCR analysis revealed that the GF2 compound caused a significant increase in the expression of FYN after 24-hour incubation in AsPC-1 cells (Fig. 2B). In case of PANC-1 cells, we have not observed any statistically significant changes after 24-hour of incubation with GF2 (Fig. 2A). In turn, after the next 48 hours we have observed a significant decrease in expression of FYN in both tested cell lines. For the GF1 compound, we have noticed a decrease in the level of FYN in PANC-1 cells after 24-hour incubation. Then, after the next 48 hours of incubation, the decreased level of FYN are maintained in both cell lines. We have observed similar changes in protein levels in both pancreatic cell lines (Fig. 3B). In detail, after 24-hours of treatment with GF2 we observed an almost 3-fold increase in the expression of Fyn in PANC-1 and AsPC-1 cells. Then, after the next 48 hours, protein levels were dramatically decreased in PANC-1. For AsPC-1, we have recorded similar effects but to a lesser extent. Similarly, the decreased expression of Fyn has also been registered for the GF1 compound in PANC-1 cells. The time-dependent changes in the expression of Fyn at the cellular level could be explained by several factors. The intensified accumulation of this protein may be the result of enhanced transcription of the FYN gene. In addition, this effect may be due to the interaction of glycofullerenes with a kinase, which may contribute to the inhibition of activity and blocking Fyn protein 


\section{CELL CYCLE}

\section{A. PANC-1}

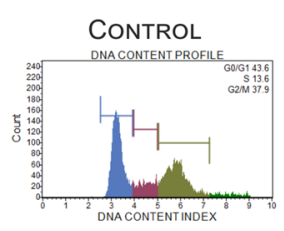

\section{B. AsPC-1}

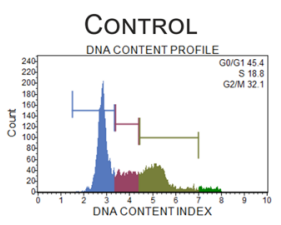

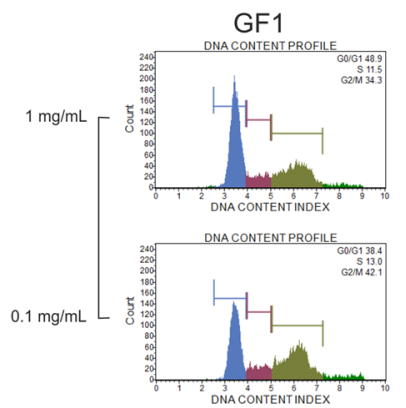

GF1

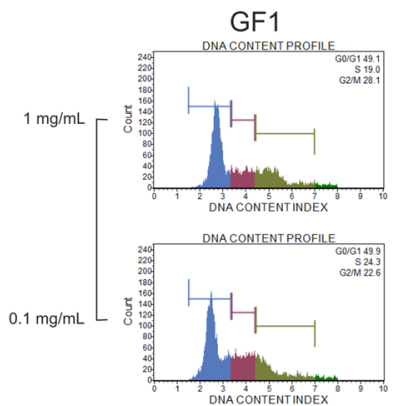

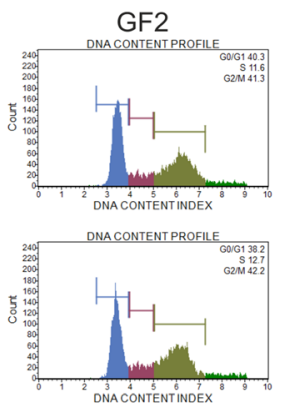

GF2

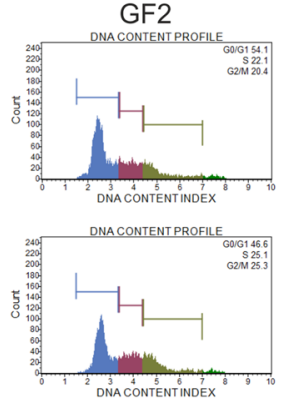

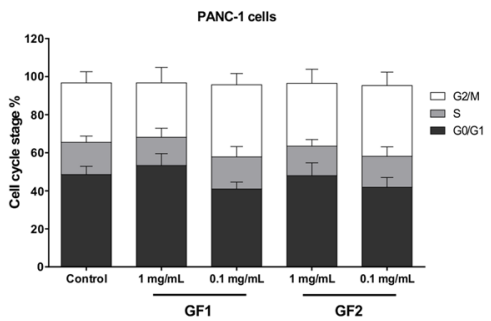

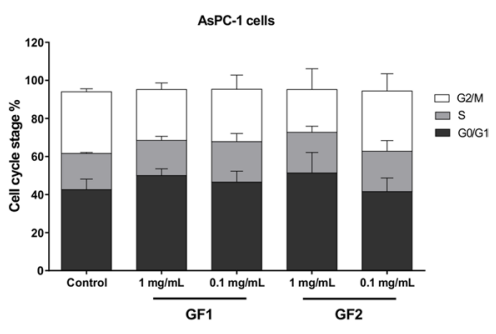

Figure 4. Influence of glycofullerenes on the cell cycle progression in pancreatic cell lines. The representative histograms from flow cytometry show cell cycle distribution after 24-hour incubation with glycofullerenes $(0.1$ and $1 \mathrm{mg} / \mathrm{mL}$ ) in PANC-1 (A) and AsPC-1 (B) cells. The graph shows the mean \pm SD percentage of cells in the G0/G1, S, and G2/M phases of the cell cycle from three independent experiments.

phosphorylation. In turn, the decrease in the level of protein over time may be indirectly related to its degradation. It should be emphasized that both carbon nanomaterials have shown the highest inhibitory potential against Fyn kinase in both water and DMEM medium. In the case of the LCK gene, we have observed the reverse behavior after treatment with carbon nanomaterials (Fig. 2). Only the GF1 compound affects changes in the LCK level in pancreatic cells. After 24 hours of treatment, we observed a significant increase in the level of this gene in PANC-1, while after 72 hours we noticed the opposite situation, namely the level of LCK had decreased in both cell lines. It is worth mentioning that the GF1 compound has exhibited greater potential for inhibiting Lck kinase (Table 1). However, on the protein level we have recorded a dramatic decrease after 24-hour incubation with GF2 in PANC-1 cells (Fig. 3B). In other cases, the Lck protein level was undetectable with the use of the Western Blot method.

The Fyn and Lck kinases belong to the Src family that plays a crucial role in the signaling networks involved in the regulation of various cellular processes, such as proliferation, cell cycle progression, adhesion, migration, invasion, and cell survival ${ }^{25-28}$. With this in mind, we have determined the influence of the tested glycofullerenes on the expression of $\mathrm{p} 21$ and $\mathrm{p} 53$ proteins that are associated with regulation of the cellular proliferation and programmed cell death induction (Fig. 3). Interestingly, we have not observed any changes in p53 expression in PANC-1. It is noteworthy that AsPC-1 cells possess two mutated p53 alleles, resulting in absence of both transcript and protein ${ }^{29}$. On the other hand, the western blot analysis has revealed influence of glycofullerenes as kinase inhibitors on upregulation of p21 protein. In general, we have observed a significant increase of p21 levels in AsPC-1 cells after 24-hour incubation with both glycofullerene compounds. We have also recorded increased p21 expression in PANC-1 cells after a 24-hour treatment with GF2. These observations are consistent with the previous report on the direct relation between inhibition of Fyn and activation of p 21 in pancreatic cells ${ }^{26}$. In addition, Jiang et al. demonstrated that inhibition of Fyn activity contributes to phosphorylation of hnRNP E1 through activation of $\mathrm{p} 21$, which affects alternative splicing of integrin $\beta$. It is noteworthy that integrins play an important role as receptors in the invasion and metastasis of pancreatic cancer cells ${ }^{30}$. On the other hand, the fact that synthesized glycofullerenes did not affect cell cycle arrest in pancreatic cells is surprising (Fig. 4).

We next decided to determine the effect of these carbon nanomaterials on the induction of cell death via autophagy. Interestingly, it had been described earlier that $\mathrm{NanoC}_{60}$ nanomaterial had been chemo-sensitizing in doxorubicin-resistant HeLa cells via autophagy mediated pathways ${ }^{31}$. Here, we have performed tests based on staining with anti-LC3 Alexa Fluor555 conjugated antibody indicative of autophagy and fluorescence measurement using flow cytometry. The results are presented in Fig. 5. In general, we observed a strong effect on the formation of autophagosomes and autophagy induction after a 24-hour treatment with GF2 in both pancreatic cancer cell lines that were tested. We have also calculated the autophagy induction ratio by comparison of GF2 to control cells, which was 1.9 for PANC-1 and 1.5 for AsPC-1 cells.

Interestingly, these effects were abolished after 48 hours. The possible explanation of these fluctuations could be activation of the cellular repair pathways, following an earlier cellular stress response. Luo et al. demonstrated 
A. PANC-1
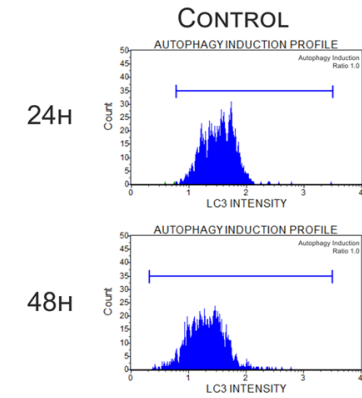

B. AsPC-1
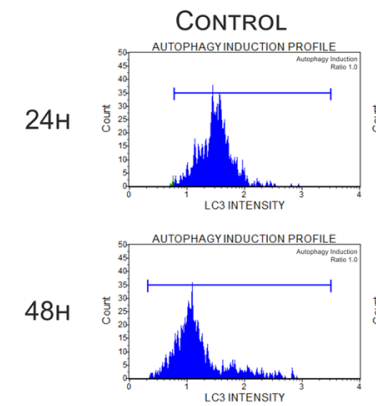
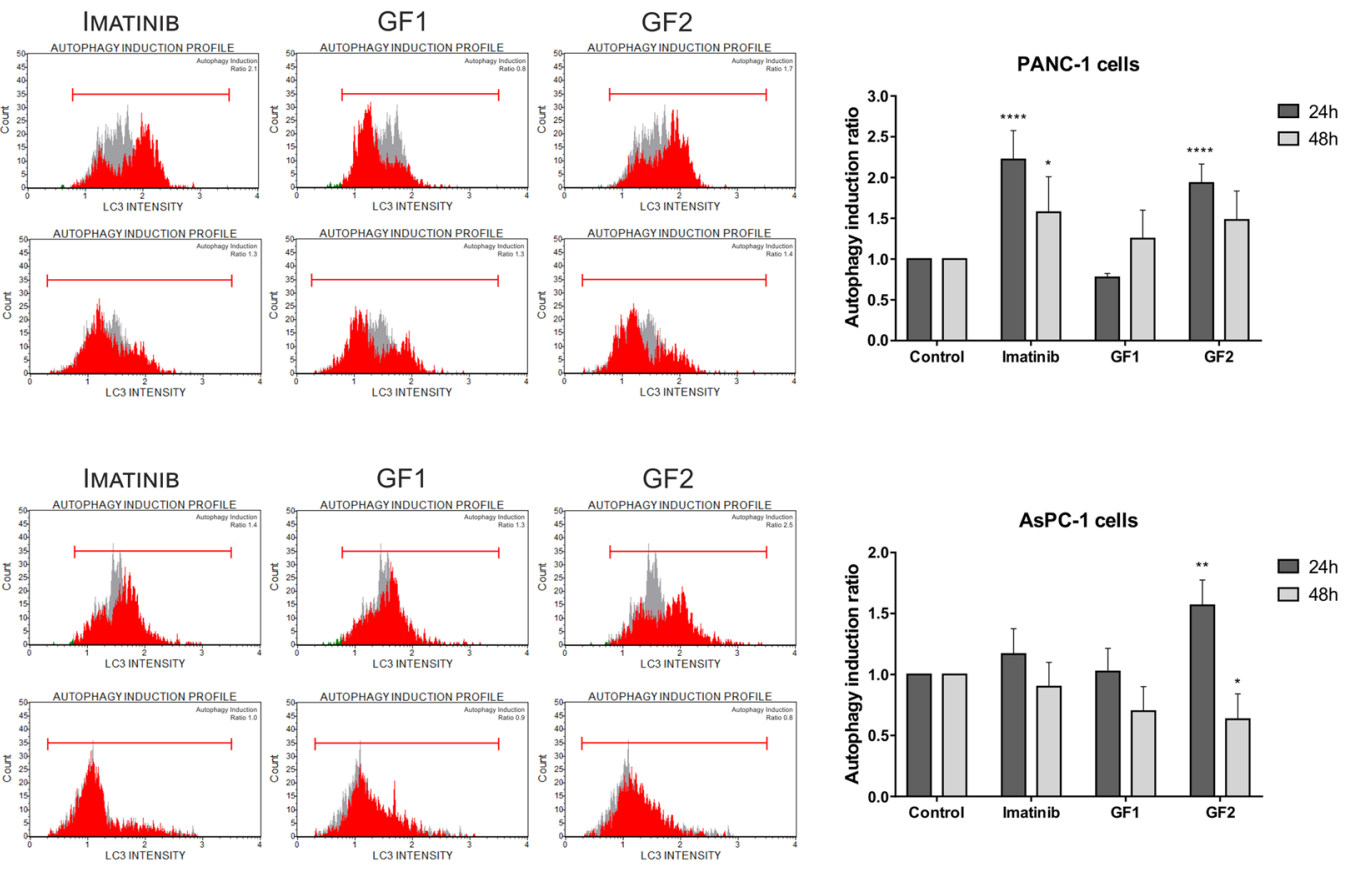

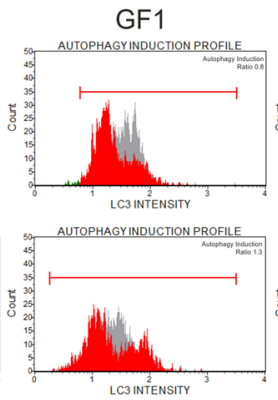

GF1

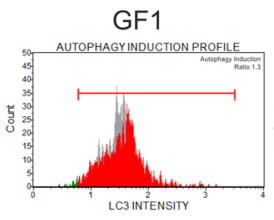

AUTOPHAGY
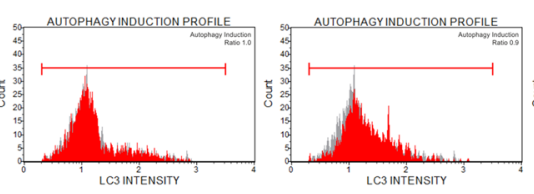

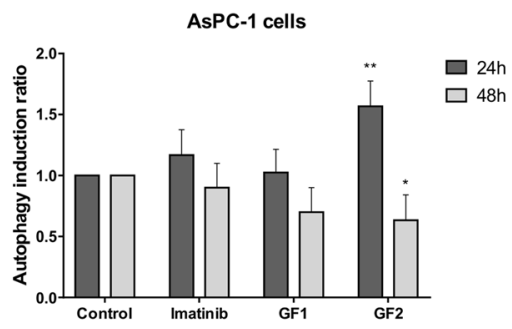

Figure 5. Influence of glycofullerenes ( $1 \mathrm{mg} / \mathrm{mL})$ on autophagy induction in PANC-1 (A) and AsPC-1 (B) cells. The histograms show the autophagy induction ratio that was calculated by comparison of fluorescence from control cells (blue or gray peak) to the tested compounds (red peak). As a positive control, Imatinib $(25 \mu \mathrm{M})$ was used. Data were analyzed using one-way ANOVA with Bonferroni's post-hoc test: $* \mathrm{p}<0.05,{ }^{*} \mathrm{p}<0.01$, $* * * \mathrm{p}<0.001$ compared to the control (untreated cells).

that autophagy may be induced by ROS formation and upregulation of $\mathrm{p} 21$ protein $^{32}$. Additionally, the sustained high level of intracellular ROS may cause activation of p38 MAPK kinase and its downstream pathways that are involved in cell growth arrest and apoptosis induction specifically in response to oxidative stress ${ }^{33,34}$. With this in mind, we decided to determine the effect of the tested glycofullerenes on the generation of ROS. In this purpose, we conducted a series of experiments with different incubation times (9, 12, 24h) with GF1, GF2 and their combination with ROS quencher - Neocuproine (NCP) in PANC-1 and AsPC-1 cells. The concentration of ROS were measured using the CellROX fluorescence assay according to protocols established by our group for small-molecule compounds ${ }^{35}$. In general, we observed an increase of ROS levels after incubation with the tested glycofullerenes in both cell lines (see Supporting Information, Fig. S10). The highest increase was observed after $9 \mathrm{~h}$ incubation in PANC-1 cells (about 130\%) - Fig. S10A. Then, in subsequent incubation time points with GF1 and GF2, we noticed a slight decrease to $110 \%$ in comparison to untreated control. Similarly effect, but to a lesser extent, was observed in AsPC-1 cells (Fig. S10B). Moreover, the addition of NCP, which is known ROS scavenger and glycofullerenes resulted in decrease ROS concentration below basal level in pancreatic cells. The upregulation of autophagy by carbon nanomaterials may also represent an interesting tool to induce cell death in cancer cells.

Heme oxygenase (HO-1) is another oxidative stress marker that may be activated by similar stimuli including polyhydroxylated fullerenes ${ }^{36}$. Under normal conditions, HO- 1 expression is low in the majority of cells and tissues. Some reports have demonstrated that upregulation of HO-1 is regulated by signaling pathways, in which MAPK kinases play a key role ${ }^{37,38}$. With this in mind, we have evaluated $\mathrm{HO}-1$ levels after 24- and 72-hour treatment with glycofullerenes (Fig. 3B). We noticed an almost 7-fold increase in the expression of HO-1 in PANC-1 cells after 72 hours in both compounds. However, a shorter incubation has no significant effect on the protein level in this cell line. Contrarily, we recorded a significant increase for GF1 after 24-hour incubation in AsPC-1 cells. With GF2 we observed a very slight increase in HO-1 levels. After a 72-hour incubation, the protein level was undetectable with the use of the Western Blot method, which may indicate that redox balance has been recovered.

To conclude, this novel report indicates glycofullerene-based inhibitors of non-receptor tyrosine kinases are able to selectively modulate the activity of Fyn A and BTK/Lck proteins. Positive influence of serum proteins may suggest that formation of fullerene-protein corona is an important factor in the process of inhibition. The performed SDS-PAGE electrophoresis studies confirmed formation of protein corona on glycofullerene GF2. Interestingly, the synthesized carbon nanomaterials have been found to be non-toxic, neutral for the pancreatic cancer cell cycle, but induces autophagy and disrupts redox balance. This work opens a new avenue in [60] fullerene-protein biomedical studies and it reflects a significant advancement in developing pancreatic cancer nanotherapeutics. Further studies explaining mechanisms of inhibition, especially for the Fyn A and BTK/Lck kinases, should be carried out with particular emphasis on determination of the $K_{m}$ and $V_{\max }$ parameters that are crucial for understanding this type of inhibition. 


\begin{abstract}
Methods
Synthesis of glycofullerenes GF1 and GF2 and their malonic acid precursors. All compounds used were reagent grade or better, solvents were used as received unless otherwise specified. The following reagents were used as received: $\mathrm{C}_{60}(99.5+\%$, SES Research, USA), D-glucosamine hydrochloride (Sigma Aldrich), DBU (1,8-diaza-bicyclo[5.4.0] undec-7-ene, Sigma Aldrich), ethyl hydrogen malonate (Sigma Aldrich), $\mathrm{CBr}_{4}$ (Sigma Aldrich), 2-amino-1,3-propanediol (AK Scientific), DIC ( $N, N$ '-Diisopropylcarbodiimide, Sigma Aldrich) and 1-hydroxybenzotriazole monohydrate (Sigma Aldrich). The following reagents: $\mathrm{Et}_{3} \mathrm{~N}$ (Acros Organics), acetic anhydride (Fisher), pyridine (Sigma Aldrich) and DMF (Sigma Aldrich) were prepared according to literature procedures by distillation with calcium hydride and used immediately. Nuclear magnetic resonance spectra were measured on a Bruker Avance III $500 \mathrm{MHz}$ NMR Spectrometer with TMS as an internal standard. MS spectra for water-insoluble compounds were collected using an Autoflex II MALDI-TOF mass spectrometer, and for water-soluble [60]fullerene derivatives by an MS electrospray ionization time-offlight (ESI-microTOF) mass spectrometer, both instruments from Bruker Daltonics Inc (Fremont, CA). High resolution spectra were performed using Shimadzu IT-ToF LC-MS System and flash chromatography was performed using Isolera Flash Purification System The purity of all compounds was assessed using a Agilent 1260 equipped with a DAAD detector at $260 \mathrm{~nm}$, RP-column: Eclipse plus C18 $(3,5 \mu \mathrm{m})$; flow $0.5 \mathrm{~mL} / \mathrm{min}$. The AFM experiments were performed using ambient pressure atomic force microscopy (AFM) NanoWizard3 from JPK Instruments. The surface topography and morphology were obtained using ultra-sharp tip ( $1 \mathrm{~nm}$ tip apex) well suitable for high resolution soft tapping mode. The data was analyzed using Gwyddion package ${ }^{39}$. The distributions of aggregates sizes were obtained by using masks on the topography maps, calculating the equivalent radius of observed.

Synthetic protocol for preparation of two [60]fullerene nanomaterials GF1 and GF2 is depicted on Fig. S1. The malonates (1) and (2) as well as glycofullerene GF2 were synthesized according to previously described methodology ${ }^{14}$.

Synthesis of diserinol malonate acetate (3). Diserinol malonate and its peracetylated version were synthesized on large scale by the modification of previously published synthetic protocol by Wharton and Wilson ${ }^{40}$. Briefly, 2-amino-1,3-propanediol (100 g, $1076 \mathrm{mmol})$ and dimethyl malonate $(57.4 \mathrm{~mL}, 500 \mathrm{mmol})$ were dissolved in dry isopropanol, heated with vigorous stirring at $50^{\circ} \mathrm{C}$ for 30 minutes and then stirred for 14 days at room temperature, under a nitrogen atmosphere. After that time, the white solid precipitated, was filtered off and washed with cold isopropanol. After recrystallization from isopropyl alcohol, the white solid was obtained and dried under lyophilization (m.p. $132^{\circ} \mathrm{C}$, yield $\left.92 \%\right)$. Serinol malonate $(25.0 \mathrm{~g}, 99.9 \mathrm{mmol})$ was suspended in pyridine $(100 \mathrm{~mL}$, $1240 \mathrm{mmol}$ ) at $0^{\circ} \mathrm{C}$, to which acetic anhydride $(150 \mathrm{~mL}, 1590 \mathrm{mmol})$ was added dropwise over the course of an hour. After reaching $25^{\circ} \mathrm{C}$, dissolution occurred and the solution was stirred continuously for 48 hours. At $0{ }^{\circ} \mathrm{C}$, methanol $(50 \mathrm{~mL}, 1225 \mathrm{mmol})$ was added to quench remaining acetic anhydride and the solution was stirred for 1 hour, whereupon the solvents were removed in vacuo and the residue (yellow oil) was dissolved in dichloromethane and recrystallized over 12 hours in hexane. The precipitate as white crystals was collected by filtration,
\end{abstract} washed with hexanes and dried in vacuo (m.p. $91^{\circ} \mathrm{C}$, lit. $91-92^{\circ} \mathrm{C}^{40}$ ).

Synthesis of [60]fullerene hexakis adduct (GF1). $\quad \mathrm{C}_{60}(720 \mathrm{mg}, 1.00 \mathrm{mmol})$ was dissolved in freshly distilled toluene $(800 \mathrm{~mL})$ with $\mathrm{CBr}_{4}(497 \mathrm{mg}, 1.5 \mathrm{mmol})$, and malonate $2(461 \mathrm{mg}, 1 \mathrm{mmol})$. At $25^{\circ} \mathrm{C}, 1,8$-diazabicyclo[5.4.0] undec-7-ene (DBU, $190 \mathrm{mg}, 1.25 \mathrm{mmol}$ ) in toluene was added and the reaction was allowed to proceed for 3 hours at room temperature under a nitrogen atmosphere. Flash chromatography was used (dichloromethane: methanol, 95:5; silica: Mallinckrodt, $75-250 \mu \mathrm{m}$ particles, $150 \AA$ pore size) to isolate the product. Purified peracylated [60] fullerene monoadduct $(65 \mathrm{mg}, 0.055 \mathrm{mmol})$ was dissolved in freshly distilled toluene $(150 \mathrm{~mL})$ with $\mathrm{CBr}_{4}$ (365 mg, $1.1 \mathrm{mmol})$, and peracylated diserinol malonate $(230 \mathrm{mg}, 0.55 \mathrm{mmol})$. At $25^{\circ} \mathrm{C}, 1,8$-diazabicyclo[5.4.0] undec-7-ene (DBU, $100 \mathrm{mg}, 0.66 \mathrm{mmol}$ ) in toluene was added slowly during 6 hours, and the reaction was allowed to proceed for 36 hours at room temperature under a nitrogen atmosphere. Flash chromatography was used (gradient from dichloromethane: methanol, 95:5 to 50:50; silica gel: Mallinckrodt, $75-250 \mu \mathrm{m}$ particles, $150 \AA ̊$ pore size) to isolate the protected product. Yield: $57 \mathrm{mg}(32 \%)$. Deprotection of acyl groups was performed in $18 \mathrm{~mL}$ of 1,4-dioxane with addiction of $2 \mathrm{~mL}$ of concentrated hydrochloric acid (36.5\%) and then stirred at room temperature for 5 days. After that time, the final product was purified by dialysis of an aqueous solution of 7 using a cellulose membrane (molecular weight exclusion limit $1.0 \mathrm{kDa}$; Spectrum Labs, USA) up to the point where electrical conductivity of a purified glycofullerene became nearly equal to the conductivity of distilled water, and then lyophilized.

Atomic force microscopy (AFM) studies. The investigated samples of glycofullerenes were transferred onto the atomically flat $\mathrm{Si}(001)$ surface in a form of small $(10 \mu \mathrm{l})$ droplets. The droplets contained typically $0.1 \mathrm{mg} / \mathrm{mL}$ of sample mixed with water or the DMEM medium containing $12 \% \mathrm{FBS}$. The droplets were left under ambient pressure to evaporate excess of water and subsequently measured.

Cell cultures. The cell cultures were prepared according to manufactures' protocols as well as our previously developed methodology for anti-cancer agents and [60]fullerene nanomaterials ${ }^{14,21}$. Briefly, human pancreatic cell lines PANC-1 and AsPC-1 were purchased from Sigma Aldrich. The cells were grown as monolayer cultures in Dulbecco's modified Eagle's medium or RPMI-1640 medium in $75 \mathrm{~cm}^{2}$ flasks (Nunc). DMEM for PANC-1 cells was supplemented with $12 \%$ heat-inactivated fetal bovine serum (Sigma). RPMI-1640 for AsPC-1 cells was supplemented with $10 \%$ heat-inactivated fetal bovine serum. Both media also contained a mix of antibiotics $-1 \% v / v$ of penicillin and streptomycin (Gibco). All of the cell lines were cultured under standard conditions at $37^{\circ} \mathrm{C}$ in a 
humidified atmosphere with $5 \% \mathrm{CO}_{2}$. All of the cell lines were subjected to routine mycoplasma testing using the PCR technique with specific Mycoplasma primers in order to ensure that there was no contamination.

Cytotoxicity assay. Cytotoxicity studies for synthesized glycofullerenes were performed and evaluated by the activity of mitochondrial reductases (MTS assay), according to a well-established protocols previously described by our group for small-molecules ${ }^{41,42}$.

Non-receptor tyrosine kinases inhibition in vitro. The inhibition of the tyrosine kinases by glycofullerenes were measured using Kinase Selectivity TK-2 profiling system and ADP-Glo assay (both reagents from Promega). This methodology was previously developed and described by our group for styrylquinazoline derivatives, and now extended and modified for [60] fullerene nanomaterials ${ }^{21}$. Before start experiments, the glycofullerenes being tested were dissolved in water or DMEM with $12 \%$ FBS to a concentration of $40 \mathrm{mg} / \mathrm{mL}$ (stock solution), which was used to prepare subsequent solutions in the concentration range of $0.05-2 \mathrm{mg} / \mathrm{mL}$ in a $1 \mathrm{x}$ Kinase Buffer. The results from these assays were presented as the percentage of the inhibition of tested kinases after treatment with the various concentrations of GF1 and GF2 nanomaterials. In addition, the inhibitory concentration $\left(\mathrm{IC}_{50}\right.$ ) values were calculated using GraphPad Prism 7.0 (GraphPad Software, USA, https://www. graphpad.com).

The mRNA expression of FYN and LCK kinases. A general method for analysis of mRNA expression of non-receptor kinases was described by our group for quinazoline derivatives and now extended and modified for [60]fullerene nanomaterials ${ }^{21}$. In short, after $24 \mathrm{~h}$ or $72 \mathrm{~h}$ treatment with tested glycofullerenes, total RNA was isolated from the pancreatic cells using TRIzol Reagent (Ambion) according to the supplier's protocols. Single-stranded cDNA was synthesized from $4 \mu \mathrm{g}$ of total RNA using a SuperScript IV Reverse Transcriptase kit with appropriate oligoprimers (dT) ${ }_{20}$ (both from Invitrogen). The quantitative Real-Time PCR was carried out in standard reaction volume of $20 \mu \mathrm{L}$, consisting of SsoAdvanced Universal SYBR Green Supermix (Biorad), primers mix $(0.5 \mu \mathrm{M})$ and $1 \mu \mathrm{L}$ of cDNA in CTX96 Touch Real-Time PCR Detection System (Biorad). The qRT-PCR reactions were carried out under the standard conditions: pre-denaturation $\left(95^{\circ} \mathrm{C} / 30 \mathrm{sec}\right)$, denaturation $\left(95^{\circ} \mathrm{C} / 15 \mathrm{sec}\right)$, annealing (temperature dependent on primers properties $\left./ 30 \mathrm{sec}\right)$, extension $\left(72^{\circ} \mathrm{C} / 60 \mathrm{sec}\right)$. The three last steps were repeated for 40 cycles. Data was interpretation and analyzed using the $2^{-\Delta \Delta \mathrm{CT}}$ method as described by Livak and Schmittgen ${ }^{43}$. The sequences of FYN, LCK and ACTB (reference gene) primers were designed in Primer 3 and were bought from Sigma Aldrich (see Table S1).

Basal level of the mRNA expression of GLUT-1 in pancreatic cells. Total RNA was isolated from PANC-1 and AsPC-1 cells using TRIzol Reagent (Ambion) according to the manufacturer's instructions. The cDNA synthesis and qRT-PCR reaction were performed as were described above. Target and reference primer pair sequences were designed in Primer 3 and were purchased from Sigma Aldrich (Table S2). Data was analyzed based on a comparison of the expression of the target genes to a reference gene - GAPDH, using the $2^{-\triangle \triangle C T}$ method. The experiments were performed at least four times.

Western blotting. Immunoblotting experiments were carried out using a well-established protocols previously described by our group for small-molecules ${ }^{35}$. Cellular lysates were collected after $24 \mathrm{~h}$ or $72 \mathrm{~h}$ treatment with glycofullerenes $(1 \mathrm{mg} / \mathrm{mL})$ in PANC-1 and AsPC-1 cell lines. The quantitation of protein was measured using a Micro BCA Protein Assay Kit (Thermo Scientific) according to the supplier's manuals. The proteins $(20 \mu \mathrm{g})$ were separated on SDS-Page gels and electrotransferred onto $0.2 \mu \mathrm{m}$ pore-size nitrocellulose membranes. After blocking step, the nitrocellulose membranes with proteins were incubated with specific primary antibodies: Fyn, Lck, p21, p53, HO-1, vinculin and GAPDH (all diluted 1:1000) overnight, then washed and incubated with horseradish peroxidase-conjugated secondary antibodies (1:1000 dilution). All primary and secondary antibodies were bought from CellSignaling. Finally, the horseradish peroxidase activity signal were detected using SuperSignal West Pico Chemiluminescent Substrate (Thermo Scientific) in ChemiDoc XRS + System (BioRad). The densitiometric analysis was performed as described elsewhere ${ }^{35}$.

Cell cycle analysis. Cell cycle analysis was carried out according to a well-established protocols previously described by our group for small-molecules ${ }^{35}$. Briefly, PANC-1 and AsPC- 1 cells were seeded according to standard procedures. Then, freshly prepared solutions of glycofullerenes at $1 \mathrm{mg} / \mathrm{mL}$ concentration were added in $3 \mathrm{~cm}$ Petri dishes (Nunc). The changes of cell cycle of pancreatic cells were determined after a 24 hour treatment with tested glycofullerenes using a Muse Cell-Cycle Kit (Millipore) according to the manufacturer's protocols.

Autophagy induction assay. PANC-1 and AsPC- 1 cells were seeded in 96-well plates (Nunc) at a density of 20,000 cells/well (for $24 \mathrm{~h}$ assay) and 10,000 cells/well (for $48 \mathrm{~h}$ assay) and incubated at $37^{\circ} \mathrm{C}$ for 24 hours. Then, the medium was removed and freshly prepared solutions of glycofullerenes at $1 \mathrm{mg} / \mathrm{mL}$ concentration were added and the cells were incubated for 24 or 48 hours. As positive control, cells were treated with Imatinib $(25 \mu \mathrm{M})$ for 24 hours. After treatment, assays were performed using a MuseAutophagy LC3-antibody based kit (Millipore) according to the manufacturer's instructions. Briefly, cells were collected, washed with cold HBSS and centrifuged at $300 \mathrm{~g}$ for $5 \mathrm{~min}$. Afterwards, cells were resuspended in $95 \mu \mathrm{L}$ of $1 \mathrm{X}$ Autophagy Reagent B with $5 \mu \mathrm{L}$ of Anti-LC3 Alexa Fluor 555 antibody and incubated for 30 minutes on ice in the dark. After this time, the cells were centrifuged and resuspended in $200 \mu \mathrm{L}$ of $1 \mathrm{X}$ Assay Buffer. Then, the cells immediately were processed for autophagy induction analysis using a Muse Cell Analyzer (Millipore). Autophagy induction ratio were calculated based on the ratio between the target sample fluorescence versus the control sample. The experiments were performed at least three times. 
ROS formation. The ROS measurements were performed according to protocols described previously by our group ${ }^{35}$. In short, PANC-1 and AsPC-1 cells were seeded according to standard procedures. Prior the experiments, the fresh solutions of glycofullerenes at $1 \mathrm{mg} / \mathrm{mL}$ concentration were prepared, which were then transferred into plate and incubated for 9, 12 and 24 hours in a kinetic experiment. For quenching reactive oxygen species, Neocuproine at $50 \mu \mathrm{M}$ concentration was added. The ROS formation was determined using a CellROX Green Reagent (Molecular Probes) according to the supplier's protocols.

Protein corona assay. Preparation of protein corona and assessments of nanomaterials interactions with FBS proteins were performed according to Strojan et al. ${ }^{44}$, with some modifications. In short, GF2 glycofullerene was prepared to a final concentration $2 \mathrm{mg} / \mathrm{mL}$ or $1 \mathrm{mg} / \mathrm{mL}$ in the $100 \%$ FBS solution and $1 \mathrm{mg} / \mathrm{mL}$ in RPMI medium supplemented with $10 \%$ FBS. Afterwards, FBS-GF2 mixtures were incubated for $2-3 \mathrm{~h}$ in $37^{\circ} \mathrm{C}$. Then, the samples (FBS-GF2) and control (100\% FBS) were centrifuged at $15,000 \times \mathrm{g}$ for 20 minutes at $4^{\circ} \mathrm{C}$, supernatants were removed and pellets were re-suspended in $1 \mathrm{~mL}$ cold PBS without $\mathrm{CaCl}_{2}$ and $\mathrm{MgCl}_{2}$. This washing procedure was repeated three times to remove non-bounded proteins. After last centrifugation, samples were re-suspended in NuPAGE LDS Sample Buffer, then vortexed and heated for $5 \mathrm{~min}$ at $95^{\circ} \mathrm{C}$. At this stage, FBS-GF2 complexes were broken down, and GF2 were removed by centrifugation at $15,000 \times \mathrm{g}$ for $20 \mathrm{~min}$. The equal amounts of the remaining proteins, which interacted with glycofullerene were separated on SDS-PAGE gels. After electrophoresis, the gels were stained with Coomassie Stain G-250 reagent for $30 \mathrm{~min}$. The image were acquired using a ChemiDoc XRS + System (BioRad) and densitometric analysis was performed as described elsewhere ${ }^{35}$.

Statistical analysis. Results are expressed as the mean \pm standard deviation (SD) from at least three independent experiments. Statistical differences in the expression of genes and proteins, autophagy induction and progression of cell cycle were calculated using the one-way ANOVA with a Bonferroni post-hoc test. A p-value of 0.05 or less was considered to be statistically significant. GraphPad Prism 7.0 software (GraphPad Software, USA, https://www.graphpad.com) was used for analysis.

Received: 7 August 2019; Accepted: 23 December 2019;

Published online: 14 January 2020

\section{References}

1. Kamisawa, T., Wood, L. D., Itoi, T. \& Takaori, K. Pancreatic cancer. Lancet 388, 73-85, https://doi.org/10.1016/S01406736(16)00141-0 (2016).

2. Gillen, S., Schuster, T., Meyer zum Büschenfelde, C., Friess, H. \& Kleeff, J. Preoperative/Neoadjuvant Therapy in Pancreatic Cancer: A Systematic Review and Meta-analysis of Response and Resection Percentages. PLOS Med. 7, e1000267, https://doi.org/10.1371/ journal.pmed.1000267 (2010)

3. Chiorean, E. G. \& Coveler, A. L. Pancreatic cancer: optimizing treatment options, new, and emerging targeted therapies. Drug. Des. Devel Ther. 9, 3529-3545, https://doi.org/10.2147/DDDT.S60328 (2015).

4. Kang, S.-g. et al. Molecular mechanism of pancreatic tumor metastasis inhibition by $\mathrm{Gd}_{8} \mathrm{C}_{82}(\mathrm{OH})_{22}$ and its implication for de novo design of nanomedicine. 109, 15431-15436, https://doi.org/10.1073/pnas.1204600109\% J Proceedings of the National Academy of Sciences (2012)

5. Zhao, R. et al. Photothermal Effect Enhanced Cascade-Targeting Strategy for Improved Pancreatic Cancer Therapy by Gold Nanoshell@Mesoporous Silica Nanorod. ACS Nano 11, 8103-8113, https://doi.org/10.1021/acsnano.7b02918 (2017).

6. Guo, Y. et al. Photothermal ablation of pancreatic cancer cells with hybrid iron-oxide core gold-shell nanoparticles. Int. J. Nanomed. 8, 3437-3446, https://doi.org/10.2147/IJN.S47585 (2013).

7. Meng, H. et al. Use of a Lipid-Coated Mesoporous Silica Nanoparticle Platform for Synergistic Gemcitabine and Paclitaxel Delivery to Human Pancreatic Cancer in Mice. ACS Nano 9, 3540-3557, https://doi.org/10.1021/acsnano.5b00510 (2015).

8. Zhao, X. et al. Co-delivery of HIF $1 \alpha$ siRNA and gemcitabine via biocompatible lipid-polymer hybrid nanoparticles for effective treatment of pancreatic cancer. Biomaterials 46, 13-25, https://doi.org/10.1016/j.biomaterials.2014.12.028 (2015).

9. Wu, P., Nielsen, T. E. \& Clausen, M. H. Small-molecule kinase inhibitors: an analysis of FDA-approved drugs. Drug. Discovery Today 21, 5-10, https://doi.org/10.1016/j.drudis.2015.07.008 (2016).

10. Moore, M. J. et al. Erlotinib plus gemcitabine compared with gemcitabine alone in patients with advanced pancreatic cancer: a phase III trial of the National Cancer Institute of Canada Clinical Trials Group. J. Clin. Oncol. 25, 1960-1966, https://doi.org/10.1200/ JCO.2006.07.9525 (2007).

11. Yezhelyev, M. V. et al. Inhibition of SRC tyrosine kinase as treatment for human pancreatic cancer growing orthotopically in nude mice. Clin. Cancer Res. 10, 8028-8036, https://doi.org/10.1158/1078-0432.CCR-04-0621 (2004).

12. Zakharian, T. Y., Balaji Sitharaman, A. S., Gilbert, B. E., Knight, V. \& Wilson, L. J. A Fullerene-Paclitaxel Chemotherapeutic: Synthesis, Characterization, and Study of Biological Activity in Tissue Culture. J. Am. Chem. Soc. 127, 12508-12509, https://doi. org/10.1021/ja0546525 (2005).

13. Lapin, N. A. et al. The effects of non-invasive radiofrequency electric field hyperthermia on biotransport and biodistribution of fluorescent [60]fullerene derivative in a murine orthotopic model of breast adenocarcinoma. J. Controlled Rel. 260, 92-99, https:// doi.org/10.1016/j.jconrel.2017.05.022 (2017).

14. Serda, M. et al. Development of photoactive Sweet-C60 for pancreatic cancer stellate cell therapy. Nanomed. (Lond.) 13, 2981-2993, https://doi.org/10.2217/nnm-2018-0239 (2018).

15. Ito, H., Duxbury, M., Zinner, M. J., Ashley, S. W. \& Whang, E. E. Glucose transporter-1 gene expression is associated with pancreatic cancer invasiveness and MMP-2 activity. Surgery 136, 548-556, https://doi.org/10.1016/j.surg.2004.05.032 (2004).

16. Isobe, H., Mashima, H., Yorimitsu, H. \& Nakamura, E. Synthesis of Fullerene Glycoconjugates through Sulfide Connection in Aqueous Media. Org. Lett. 5, 4461-4463, https://doi.org/10.1021/ol0357705 (2003).

17. Raoof, M., Mackeyev, Y., Cheney, M. A., Wilson, L. J. \& Curley, S. A. Internalization of C60 fullerenes into cancer cells with accumulation in the nucleus via the nuclear pore complex. Biomaterials 33, 2952-2960, https://doi.org/10.1016/j. biomaterials.2011.12.043 (2012).

18. Heimann, J., Morrow, L., Anderson, R. E. \& Barron, A. R. Understanding the relative binding ability of hydroxyfullerene to divalent and trivalent metals. Dalton Trans. 44, 4380-4388, https://doi.org/10.1039/C4DT03376K (2015).

19. Lee, J. et al. Photochemical and antimicrobial properties of novel C60 derivatives in aqueous systems. Environ. Sci. \& Technol. 43, 6604-6610 (2009). 
20. Mackeyev, Y., Mark, C., Kumar, N. \& Serda, R. E. The influence of cell and nanoparticle properties on heating and cell death in a radiofrequency field. Acta biomaterialia 53, 619-630 (2017).

21. Mularski, J., Malarz, K., Pacholczyk, M. \& Musiol, R. The p53 stabilizing agent CP-31398 and multi-kinase inhibitors. Designing, synthesizing and screening of styrylquinazoline series. Eur. J. Med. Chem. 163, 610-625, https://doi.org/10.1016/j. ejmech.2018.12.012 (2019).

22. Calvaresi, M. \& Zerbetto, F. Baiting Proteins with C60. ACS Nano 4, 2283-2299, https://doi.org/10.1021/nn901809b (2010).

23. Ke, P. C., Lin, S., Parak, W. J., Davis, T. P. \& Caruso, F. A Decade of the Protein Corona. ACS Nano 11, 11773-11776, https://doi. org/10.1021/acsnano.7b08008 (2017).

24. Zhang, T.-X., Zhu, G.-Y., Lu, B.-Y., Zhang, C.-L. \& Peng, Q. Concentration-dependent protein adsorption at the nano-bio interfaces of polymeric nanoparticles and serum proteins. Nanomedicine 12, 2757-2769 (2017).

25. Parsons, S. J. \& Parsons, J. T. Src family kinases, key regulators of signal transduction. Oncogene 23, 7906, https://doi.org/10.1038/ sj.onc.1208160 (2004).

26. Chen, Z.-Y. et al. Roles of Fyn in pancreatic cancer metastasis. J. Gastroenterology Hepatology 25, 293-301, https://doi.org/10.1111/ j.1440-1746.2009.06021.x (2010).

27. Yadav, V. \& Denning, M. F. Fyn is induced by Ras/PI3K/Akt signaling and is required for enhanced invasion/migration. Mol. Carcinogenesis 50, 346-352, https://doi.org/10.1002/mc.20716 (2011)

28. Je, D. W., O, Y. M., Ji, Y. G., Cho, Y. \& Lee, D. H. The Inhibition of Src Family Kinase Suppresses Pancreatic Cancer Cell Proliferation. Migration, Invasion. 43, 768-776, https://doi.org/10.1097/mpa.0000000000000103 (2014).

29. Berrozpe, G., Schaeffer, J., Peinado, M. A., Real, F. X. \& Perucho, M. Comparative analysis of mutations in the p53 and K-ras genes in pancreatic cancer. Int. J. Cancer 58, 185-191, https://doi.org/10.1002/ijc.2910580207 (1994).

30. Jiang, P., Li, Z., Tian, F., Li, X. \& Yang, J. Fyn/heterogeneous nuclear ribonucleoprotein E1 signaling regulates pancreatic cancer metastasis by affecting the alternative splicing of integrin beta1. Int. J. Oncol. 51, 169-183, https://doi.org/10.3892/ijo.2017.4018 (2017).

31. Zhang, Q. et al. Autophagy-mediated chemosensitization in cancer cells by fullerene C60 nanocrystal. Autophagy 5, 1107-1117, https://doi.org/10.4161/auto.5.8.9842 (2009).

32. Luo, Y. et al. Autophagy regulates ROS-induced cellular senescence via p21 in a p38 MAPK $\alpha$ dependent manner. Exp. Gerontology 46, 860-867, https://doi.org/10.1016/j.exger.2011.07.005 (2011).

33. Yang, S.-t et al. Carbon Dots for Optical Imaging in Vivo. J. Am. Chem. Soc. 131, 11308-11309, https://doi.org/10.1021/ja904843x (2009).

34. Dolado, I. et al. p38 $\alpha$ MAP Kinase as a Sensor of Reactive Oxygen Species in Tumorigenesis. Cancer Cell 11, 191-205, https://doi. org/10.1016/j.ccr.2006.12.013 (2007).

35. Mrozek-Wilczkiewicz, A., Malarz, K., Rejmund, M., Polanski, J. \& Musiol, R. Anticancer activity of the thiosemicarbazones that are based on di-2-pyridine ketone and quinoline moiety. Eur. J. Medicinal Chem. 171, 180-194, https://doi.org/10.1016/j. ejmech.2019.03.027 (2019).

36. Ye, S. et al. Polyhydroxylated fullerene attenuates oxidative stress-induced apoptosis via a fortifying Nrf2-regulated cellular antioxidant defence system. Int. J. Nanomed. 9, 2073-2087, https://doi.org/10.2147/IJN.S56973 (2014).

37. Paine, A., Eiz-Vesper, B., Blasczyk, R. \& Immenschuh, S. Signaling to heme oxygenase-1 and its anti-inflammatory therapeutic potential. Biochemical Pharmacology 80, 1895-1903, https://doi.org/10.1016/j.bcp.2010.07.014 (2010).

38. Yang, X. et al. Cyclooxygenase2mediated upregulation of heme oxygenase 1 mitigates the toxicity of deuteriumtritium fusion radiation. Int. J. Mol. Med. 42, 1945-1954, https://doi.org/10.3892/ijmm.2018.3799 (2018).

39. Nečas, D. \& Klapetek, P. Gwyddion: an open-source software for SPM data analysis. Open. Phys. 10, 181-188 (2012).

40. Wharton, T. \& Wilson, L. J. Highly-Iodinated Fullerene as a Contrast Agent For X-ray Imaging. Bioorganic \& Medicinal Chem. 10, 3545-3554, https://doi.org/10.1016/S0968-0896(02)00252-3 (2002).

41. Serda, M. et al. Exploring the Anti-Cancer Activity of Novel Thiosemicarbazones Generated through the Combination of RetroFragments: Dissection of Critical Structure-Activity Relationships. PLOS ONE 9, e110291, https://doi.org/10.1371/journal. pone.0110291 (2014).

42. Pastuch-Gawołek, G. et al. Small molecule glycoconjugates with anticancer activity. Eur. J. Medicinal Chem. 112, 130-144, https:// doi.org/10.1016/j.ejmech.2016.01.061 (2016).

43. Livak, K. J. \& Schmittgen, T. D. Analysis of relative gene expression data using real-time quantitative PCR and the $2^{-\Delta \Delta C T}$ method. methods 25, 402-408 (2001)

44. Strojan, K. et al. Dispersion of nanoparticles in different media importantly determines the composition of their protein corona. PLoS one 12, e0169552 (2017).

\section{Acknowledgements}

Dr Maciej Serda and Dr Katarzyna Malarz thank National Science Center (Poland) for the support (SONATA UMO-2016/23/D/NZ7/00912 and PRELUDIUM UMO-2016/23/N/NZ7/00351 grants). All authors thanks prof. Lon J. Wilson (Rice University, USA) and prof. Stuart J. Corr (Baylor College of Medicine, USA) for the fruitful discussions during the process of glycofullerene designing and synthesis.

\section{Author contributions}

Conception and design: M.S., K.M., A.M.W., R.M. and S.A.C. Acquisition of data: M.S., K.M., A.M.W. and M.W. Analysis and interpretation of the data: M.S., K.M., M.W., A.M.W., R.M. Manuscript preparation: M.S. and K.M. wrote the manuscript. All authors read and approved the final manuscript.

\section{Competing interests}

The authors declare no competing interests.

\section{Additional information}

Supplementary information is available for this paper at https://doi.org/10.1038/s41598-019-57155-7.

Correspondence and requests for materials should be addressed to M.S.

Reprints and permissions information is available at www.nature.com/reprints.

Publisher's note Springer Nature remains neutral with regard to jurisdictional claims in published maps and institutional affiliations. 
(c) (i) Open Access This article is licensed under a Creative Commons Attribution 4.0 International License, which permits use, sharing, adaptation, distribution and reproduction in any medium or format, as long as you give appropriate credit to the original author(s) and the source, provide a link to the Creative Commons license, and indicate if changes were made. The images or other third party material in this article are included in the article's Creative Commons license, unless indicated otherwise in a credit line to the material. If material is not included in the article's Creative Commons license and your intended use is not permitted by statutory regulation or exceeds the permitted use, you will need to obtain permission directly from the copyright holder. To view a copy of this license, visit http://creativecommons.org/licenses/by/4.0/.

(C) The Author(s) 2020 